\title{
Electron relaxation and mobility in the inverted band quantum well $\mathrm{CdTe} / \mathrm{Hg}_{1-\mathrm{x}} \mathrm{Cd}_{\mathrm{x}} \mathrm{Te} / \mathrm{CdTe}$
}

\author{
Ye.O. Melezhik*, J.V. Gumenjuk-Sichevska**, F.F. Sizov \\ V.E. Lashkaryov Institute of semiconductor physics of NASU, \\ 03028, Nauki av., 41, Kiev, Ukraine, +38(044)-525-18-10 \\ *Corresponding authore-mail:emelezhik@gmail.com;**e-mail gumenjuk@gmail.com
}

\begin{abstract}
Electron relaxation processes at nitrogen temperatures in $\mathrm{CdTe} / \mathrm{Hg}_{1-}$ ${ }_{x} \mathrm{Cd}_{\mathrm{x}} \mathrm{Te} / \mathrm{CdTe}$ quantum well $(\mathrm{QW})$ with an inverted band structure is modelled. In this structure, scattering by longitudinal optical phonons, charged impurities, acoustic phonons and interfaces were taken into account. It was found that for undoped and lightly doped QWs (concentration of background $n$-type charged impurities in the well is $10^{14}-10^{16} \mathrm{~cm}^{-3}$ or less), for $x$ close to the band inversion value 0.16 , the electron mobility grows considerably when the QW width decreases. This mobility is higher for samples with smaller concentrations of charged impurities.
\end{abstract}

Keywords: quantum well, mobility, $\mathrm{HgCdTe}$, terahertz range.

Manuscript received 11.10.13; revised version received 15.01.14; accepted for publication 20.03.14; published online 31.03.14.

\section{Introduction}

Terahertz spectral range attracts great interest of researchers worldwide. Nowadays, terahertz detection systems are widely used not only in astronomy, but also beginning to find their application in biology, medicine and security. These areas of usage need light-weight, compact, high-speed and sensitive detectors which do not need deep cooling. Most of the existing $\mathrm{THz}$ detectors (e.g., superconducting hot electron bolometers, SIS (superconductor-insulator-superconductor) structures, etc.) have high sensitivity (noise-equivalent power $\mathrm{NEP} \sim 10^{-15}-10^{-19} \mathrm{~W} / \mathrm{Hz}^{1 / 2}$ ) and high speed (response time $\tau \sim 10^{-9}-10^{-11} \mathrm{~s}$ ) but need deep cooling up to $T=0.1-4 \mathrm{~K}$ [1]. Semiconductor bolometer InSbbased detectors have high sensitivity $\left(\mathrm{NEP} \sim 10^{-17}\right.$ $\left.\mathrm{W} / \mathrm{Hz}^{1 / 2}\right)$ within the spectral range $(\lambda \approx 1-4 \mu \mathrm{m})$. But these detectors also need deep cooling and are characterized by relatively high response time $\left(\tau \approx 10^{-6} \mathrm{~s}\right)$ [2]. Response of existing un-cooled $\mathrm{THz}$ detectors in most cases is limited by times $\tau \geq 10 \mathrm{~ms}$ (except of
Schottky barriers and FET-based THz detectors [3]) and their NEP is of the order of $10^{-9}-10^{-10} \mathrm{~W} / \mathrm{Hz}^{1 / 2}$.

Heterostructures based on the narrow band-gap semiconductors (like solid solutions of $\mathrm{Hg}_{1-\mathrm{x}} \mathrm{Cd}_{\mathrm{x}} \mathrm{Te}$ ) hold a high promise to be prospective materials for creation of $\mathrm{THz}$ detectors. High-quality $\mathrm{Hg}_{1-\mathrm{x}} \mathrm{Cd}_{\mathrm{x}} \mathrm{Te}$ quantum wells (QWs) are characterized by low effective masses of localized electrons, high electron mobilities even at temperatures $T \geq 77 \mathrm{~K}$, and possess great potential for detection of terahertz radiation.

The problem of growth of $\mathrm{Hg}_{1-\mathrm{x}} \mathrm{Cd}_{\mathrm{x}} \mathrm{Te}$ QWs and investigation of their properties is widely addressed in the literature. Photoluminescence $[4,5]$ and photoconductivity [6] of $\mathrm{Hg}_{1-\mathrm{x}} \mathrm{Cd}_{\mathrm{x}} \mathrm{Te}$ QWs grown on different substrates ( $\mathrm{Si}, \mathrm{GaAs}, \mathrm{ZnTe}, \mathrm{CdTe})$ have been studied. It is shown in [7] that high-quality HgTe QW structures can be used for all-electric detection of radiation ellipticity in a wide spectral range, from far-infrared to mid-infrared wavelengths. Measurements of electrical conductivity, the Hall coefficient, and photoluminescence of ion-milled $\mathrm{Hg}_{1-\mathrm{x}} \mathrm{Cd}_{\mathrm{x}} \mathrm{Te}$ films were performed in [8]. Recently 
existence of 2D semimetal in the quantum wells of HgTe was revealed and measurements of cyclotron resonance in this structure were performed in [9].

A lot of works are devoted to electron mobility in GaAs 2D heterostructures. In [10], limits of mobility improving in $2 \mathrm{D}$ GaAs structure were theoretically exhibited for extremely low temperatures. At $T=1 \mathrm{~K}$ the mobility achieved $10^{8} \mathrm{~cm}^{2} / \mathrm{V} \mathrm{s}$ and was limited by background impurities of the reduced concentration $10^{12} \mathrm{~cm}^{-3}$. At liquid helium temperatures, the mobility was dropped significantly and limited by acoustic phonon scattering.

There are much less experimental data on mobility in mercury-cadmium-telluride 2D structures. Mobilities were studied experimentally in SL with thin wells [11] with the direct band structure, but they had not get any mobility increasing in comparison with 3D. In [12] it was shown that inverted band structure should be realised in structures with QW thicker then $\sim 6.5 \mathrm{~nm}$.

The high mobility $\left(2.8 \times 10^{5} \mathrm{~cm}^{2} /(\mathrm{V} \cdot \mathrm{s})\right)$ was observed in the QW CdTe/HgTe/CdTe of $16 \mathrm{~nm}$ width with inverted band structure at the temperature $3 \mathrm{~K}$ [13]. On the other hand, for bulk MCT it was shown [14] that electron mobility increases in the neighborhood of composition value of 0.16 up to $10^{6} \mathrm{~cm}^{2} /(\mathrm{V} \cdot \mathrm{s})$ at the liquid nitrogen temperature.

Here, we try to define limiting mechanisms of 2D mobility in $\mathrm{CdTe} / \mathrm{HgCdTe} / \mathrm{CdTe}$ hetero structures at moderate cooling. We modeled electron relaxation processes in $\mathrm{CdTe} / \mathrm{Hg}_{1-\mathrm{x}} \mathrm{Cd}_{\mathrm{x}} \mathrm{Te} / \mathrm{CdTe} \mathrm{QWs}$ with thick wells and inverted band scheme to find the possibility to increase the in-plane mobility in these structures. Our studies are aimed to estimate the optimal QW parameters for the creation of high-speed and moderately cooled $\mathrm{THz}$ detectors, namely field-effect transistors with high mobilities in shallow channel.

\section{The $Q W$ band structure and properties}

\subsection{Band scheme and properties of $Q W$}

Inside $\mathrm{CdTe} / \mathrm{Hg}_{1-\mathrm{x}} \mathrm{Cd}_{\mathrm{x}} \mathrm{Te} / \mathrm{CdTe} \mathrm{QW}$ for compositions $0<x<0.16$, the inverted band scheme is realized [15], while the direct band scheme is realized in the barriers (Fig. 1). All calculations are carried out for the liquid nitrogen temperature $T=77 \mathrm{~K}$. In this chapter, we describe some properties of the system under consideration.

The concentration of charged impurities in these QWs can be about $10^{14}-10^{16} \mathrm{~cm}^{-3}[6,7,16]$. Due to the inverted band scheme and high density of states for heavy holes, the Fermi level lies higher than the bottom of the conduction band in the well.

For undoped and lightly doped $\mathrm{Hg}_{1-\mathrm{x}} \mathrm{Cd}_{\mathrm{x}} \mathrm{Te}$ at liquid nitrogen temperatures, there are two dominant relaxation mechanisms for localized electrons in bulk crystals - scattering by longitudinal optical (LO) phonons and scattering by charged impurities $[14,16]$. With the growth of temperature, the role of scattering by optical phonons increases, while scattering by charged impurities becomes less important. In heterostructures, additional important scattering on interface roughness appears. In this paper, we discuss all these mechanisms. Also, we estimate scattering by acoustic phonons to prove that it is minor scattering mechanism for our system.

The scattering of localized electrons in the QW with an inverted band scheme qualitatively differs from the scattering in the direct band heterostructures. In direct band semiconductors, the conduction band is formed by the levels with $\Gamma 6$ symmetry, while the lighthole and the heavy-hole bands are formed by $\Gamma 8$ levels. In semiconductors with the inverted band scheme, $Г 6$ and $\Gamma 8$ levels change their positions - and now the conduction band is formed by $\Gamma 8$ levels, the heavy-hole band has the same symmetry as the conduction band and touches the conduction band at $k=0$, while the lighthole band is formed by $\Gamma 6$ levels and lies below the conduction band.

There are contradicting experimental results in literature, which describe the valence-band offset $\Delta$ in the $\mathrm{HgTe} / \mathrm{CdTe} \mathrm{QWs}$. The most cited values are $0.35 \mathrm{eV}$ $[19,20]$ and $0.55 \mathrm{eV}$ at $\mathrm{x}=0$, they are supposed to change linearly with the composition. In our calculations, we use the value $0.55 \times(1-x) \mathrm{eV}[21,22,23]$ (see Fig. 1).

\subsection{Interface levels and wave functions}

In direct band QWs, all levels of localized electrons lie above the bottom of the conduction band. In general, the Fermi level lies in the band gap of such structures. Thus, electron levels are located much higher than the Fermi level, and their occupancy influences the electron scattering processes only slightly.

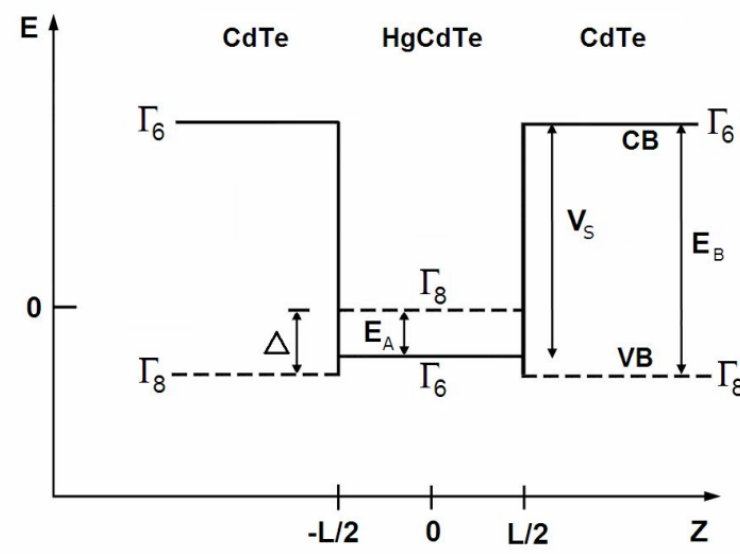

Fig. 1. Band scheme of $\mathrm{QW} \mathrm{CdTe} / \mathrm{Hg}_{1-\mathrm{x}} \mathrm{Cd}_{\mathrm{x}} \mathrm{Te} / \mathrm{CdTe}$ along the $\mathrm{z}$ axis of the $\mathrm{QW}$, where $\mathrm{L}$ is the QW width, $\Delta-$ valence band offset, $V_{S}$ - conduction band offset and $E_{A}$ - negative band gap inside the QW, $E_{B}$ - positive band gap of the barrier. $V_{S}=\left(E_{B}-\right.$ $\left.\Delta-E_{A}\right)$ 

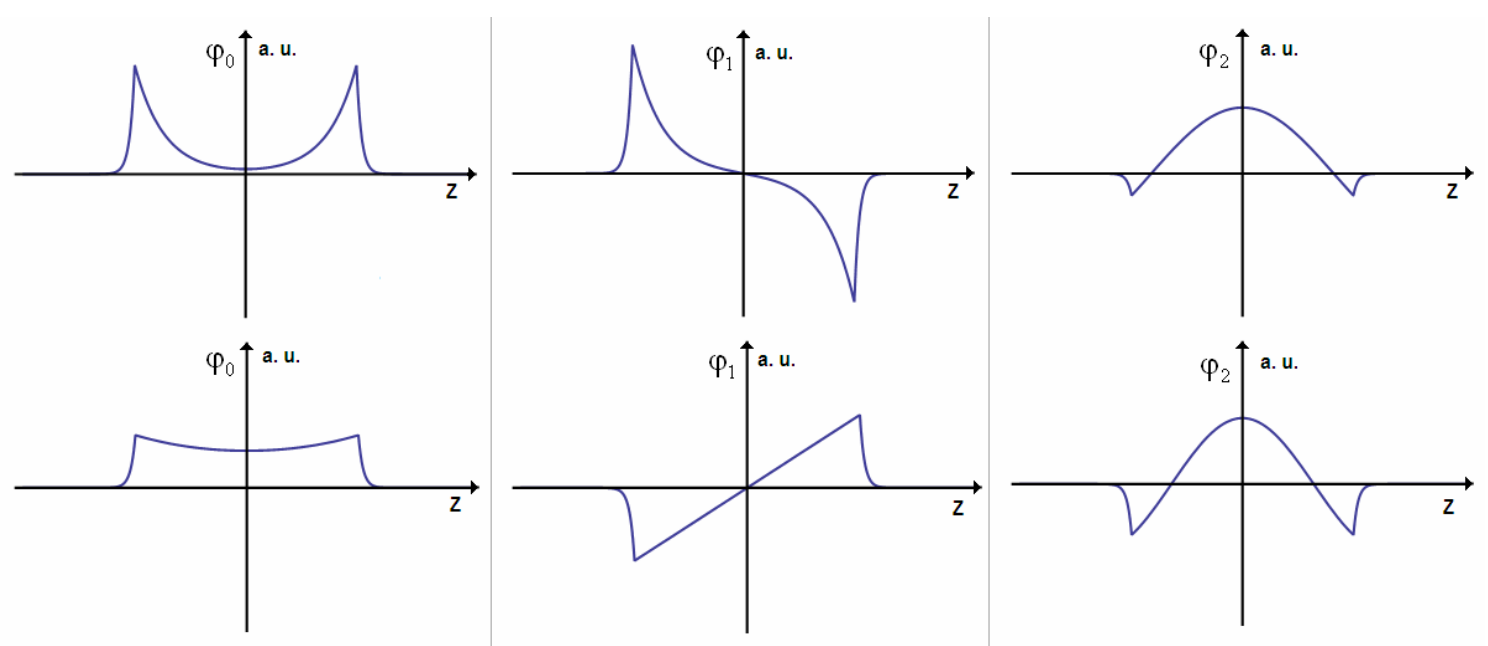

Fig. 2. Electron wave functions for the QW width $L=50 \mathrm{~nm}$. The horizontal axis corresponds to $z$ coordinate; peaks of the wave functions are at the hetero-interfaces. The vertical axis represents the magnitude of the wave function in arbitrary units (a. u.). The upper row corresponds to the composition $\mathrm{x}=0$ while the lower row corresponds to the composition $x=0.12$.

$\mathrm{CdTe} / \mathrm{Hg}_{1-\mathrm{x}} \mathrm{Cd}_{\mathrm{x}} \mathrm{Te} / \mathrm{CdTe} \mathrm{QWs}$ with the composition $0<x<0.16$ have an inverted band scheme inside the well and a direct band scheme in barriers $[15,17]$. Thus in such structures there exist one or two electron levels which lie below the bottom of conduction band of the well (see Fig. 3) due to the mixture of the states of different symmetry and due to the change of the sign of effective mass when crossing the interface [15]. These levels are built from evanescent states in each of the host layers and are localized on the interfaces (see Fig. 2). They are called "interface" levels [15] and the energy of the ground interface level reduces at small well widths [18, p. 81]. Consequently, one or several levels of localized electrons are located below the Fermi level (see Fig. 3). Such position of these levels drastically changes the occupation of them and influences the electrons scattering.

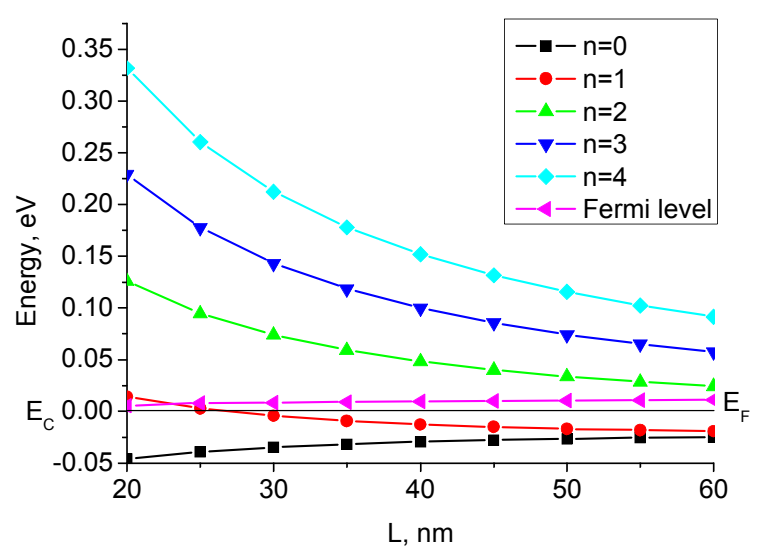

Fig. 3. Dependencies of energy spectra and Fermi level on the well width $L$. The temperature is taken to be $T=77 \mathrm{~K}$, the composition $x=0.12 . E_{C}=0$ is the bottom of the conduction band and $E_{V}=-76.6 \mathrm{meV}$ - top of the valence band in the well.
It is important to note that for compositions $\mathrm{x}$ that are close to zero, ground level electrons are localized at interfaces of the QW, which leads to the importance of interface scattering. On the other hand, ground level electrons are localized inside the quantum well for the compositions $x$ within the range $0.1<x<0.16$ (see Fig. 2 ). In this case, the probability to find electron at interfaces is low and interface scattering is also supposed to be small. In this paper, we consider $\mathrm{CdTe} / \mathrm{Hg}_{1-\mathrm{x}} \mathrm{Cd}_{\mathrm{x}} \mathrm{Te} / \mathrm{CdTe} \mathrm{QW}$, as an example, for composition $x=0.12$.

Principal features of electron relaxation in the $\mathrm{CdTe} / \mathrm{Hg}_{1-\mathrm{x}} \mathrm{Cd}_{\mathrm{x}} \mathrm{Te} / \mathrm{CdTe} \mathrm{QW}$ are determined by the influence of the ground levels occupation on the scattering processes.

For undoped QW with the composition $x=0.12$ at the temperature $T=77 \mathrm{~K}$, as shown in Fig. 3, the Fermi level is evaluated by alignment of concentrations of electrons and heavy holes. Fermi level varies from 1.8 $\mathrm{meV}$ from the $\Gamma_{8}$ band bottom of electrons in the well for QW of $20-\mathrm{nm}$ width to $8.6 \mathrm{meV}$ for $\mathrm{QW}$ of $60-\mathrm{nm}$ width. The evaluated concentration of electrons in $\mathrm{QW}$ varies from $10^{17} \mathrm{~cm}^{-3}$ for QW of $20 \mathrm{~nm}$ width to $4 \times 10^{16}$ $\mathrm{cm}^{-3}$ for QW of $60 \mathrm{~nm}$ width.

\subsection{Influence of the misfit strain on electron spectra}

To calculate the energy structure of $\mathrm{CdTe} /$ $\mathrm{Hg}_{1-\mathrm{x}} \mathrm{Cd}_{\mathrm{x}} \mathrm{Te} / \mathrm{CdTe}$ quantum wells, it is important to consider the band shift and band splitting due to the strains in the heterostructure. These strains arise from lattice mismatch between the materials of the well and the matrix. To estimate such strains, we use the simple approach that assumes that the in-plain lattice parameters of the well material are forced to be the same as appropriate parameters of the barrier material [24, 25].

Consequently, the biaxial strain in the layer plane arises inside the well $(x, y)$. The value of this strain can 
be estimated from the simple formula $e_{x x}=e_{y y}=\frac{a_{C d T e}-a_{H g C d T e}}{a_{H g C d T e}}[25,26]$, where $a_{\mathrm{CdTe}}$ and $a_{\mathrm{HgCdTe}}$ are the lattice parameters of the appropriate layers in the heterostructure. The values are as follows: $a_{\mathrm{CdTe}}=0.6482 \mathrm{~nm}, a_{\mathrm{HgTe}}=0.64605 \mathrm{~nm}$ at T=300 K [27]. For $x=0.12, a_{\mathrm{HgCdTe}}=0.64631 \mathrm{~nm}$.

With the values of the lattice parameters of the well and barrier, we get the strain components $e_{x x}=e_{y y}=$ $0.0029274, e_{z z}=0$.

According to $[28,34]$, biaxial strain leads to two effects. The first effect is the alteration of the gap value between $\Gamma_{6}$ and $\Gamma_{8}$ bands. The change of this gap is given by [29]:

$\Delta E_{g}=(C-a) \sum_{i=1}^{3} e_{i i}$

where $(C-a)$ is the difference of deformation potentials of conduction and valence bands, respectively. $(C-a)_{\mathrm{HgTe}}=-3.69 \mathrm{eV}$ [29], $(C-a)_{\mathrm{CdTe}}=-3.16 \mathrm{eV}$ [30]. For $\mathrm{Hg}_{1-\mathrm{x}} \mathrm{Cd}_{\mathrm{x}} \mathrm{Te}$ with $x=0.12$, the value of this parameter is found from the linear approximation $(C-a)_{\mathrm{HgCdTe}}=-3.63 \mathrm{eV}$. Effective band gap for $x=0.12$ is $-50.9 \mathrm{meV}$ without strain. Taking into account strain effect, the band gap reaches $-72.1 \mathrm{meV}$.

The second effect of biaxial tensile strain [31] is in splitting of electron and heavy-holes $\Gamma_{8}$ states. In this case, the top of $\Gamma_{8}$ heavy-hole band shifts under the bottom of the $\Gamma_{8}$ conduction band. The value of such splitting in the point $k=0$ is given by [28, 34]:

$\Delta E_{\Gamma_{g}}=2\left|b e_{x x}\right|$

From [32, 33], Eq. (1.2) and the Hooke's law in the simplest form we can estimate the modulus of deformation potential $b$ to be of the order of $1.54 \mathrm{eV}$. In further calculations, we assume that the difference between the estimated value of $\mathrm{b}$ for $\mathrm{HgTe}$ and the value of this potential for $\mathrm{Hg}_{1-\mathrm{x}} \mathrm{Cd}_{\mathrm{x}} \mathrm{Te}$ with the composition $x=0.12$ is negligible. The calculated splitting between light and heavy $\Delta \Gamma_{8}$ bands is about $9 \mathrm{meV}$. Thus, the strain introduces the gap between electrons and heavy holes. Consequently the gapless case can not be realized even for $x=0.16$.

\section{Boltzmann transport}

Presented in this section is the general theory that is the basis for all further calculations of electron relaxation times and mobilities. We start from the Hamiltonian of the localized electrons being scattered and use the Boltzmann transport equation (BTE) to obtain relaxation times for longitudinal optical phonon scattering and charged impurities scattering. It is assumed that external electric field is applied in the plane of QW. In our calculations, only electron-lattice interactions are taken into account while electron-electron interactions are neglected.

The Hamiltonian of localized electron can be written as:

$$
H=H_{0}+e \vec{F} \cdot \vec{r}+H_{d e f}
$$

where $H_{0}$ is unperturbed Hamiltonian, while $H_{\text {def }}$ includes the local fluctuations of the electrostatic potentials due to the defects and lattice vibrations, $\vec{F}$ is a weak, constant and homogeneous electric field. We use BTE in the simplest form:

$$
\begin{aligned}
& \frac{d f_{n}}{d t}=\frac{\partial f_{n}}{\partial t}+\frac{\hbar \vec{k}}{m_{n}} \cdot \vec{\nabla}_{r} f_{n}-\frac{e \vec{F}}{\hbar} \cdot \vec{\nabla}_{k} f_{n}= \\
& =\sum_{n^{\prime}, k_{\perp}^{\prime}} W_{n^{\prime} k_{\perp}^{\prime} \rightarrow n k_{\perp}} f_{n^{\prime}}\left(k_{\perp}^{\prime}\right)-W_{n k_{\perp} \rightarrow n^{\prime} k_{\perp}^{\prime}} f_{n}\left(k_{\perp}^{\prime}\right)
\end{aligned}
$$

where $\left(n, k_{\perp}\right)$ and $\left(n^{\prime}, k_{\perp}^{\prime}\right)$ describe the initial and final states of electron during the scattering process, $k_{\perp}$ and $k_{\perp}^{\prime}$ are two-dimensional electron wave-vectors, $f_{n}\left(k_{\perp}\right)=\left(\exp \left[\frac{E_{n}\left(k_{\perp}\right)-E_{F}}{k_{B} T}\right]+1\right)^{-1}$ is the Fermi-Dirac distribution function for localized electrons in the QW, energy $E$ depends on the wave-vector $k$ via the nonparabolic dispersion law, for example in the well this law has the form $E_{n}\left(k_{\perp}\right)=\frac{1}{2}\left(E_{A}+\sqrt{E_{A}^{2}+8 / 3\left(k_{\perp}^{2}+k_{n}^{2}\right) P^{2}}\right)$ [17], where $P$ is the Kane matrix element equal to $8.3 \times 10^{-8} \mathrm{eV} \cdot \mathrm{cm}$. Using the principle of detailed balance, one can obtain that $W_{n^{\prime} k_{\perp}^{\prime} \rightarrow n k_{\perp}}=W_{n k_{\perp} \rightarrow n^{\prime} k_{\perp}^{\prime}}$. The distribution function can be written as sum of symmetric and asymmetric parts:

$f=f^{S}+f^{A}$

The sum over the symmetric part $f^{S}$ in Eq. (2.2) is equal to zero. We deal with the homogeneous system that is in the steady state under a uniform electric field. Changing the variable in the derivative in the left-hand side of Eq. (2.2), we obtain:

$$
\begin{aligned}
& -\frac{e}{m_{n}} \hbar k_{\perp} \cdot F \frac{\partial f_{n}\left(k_{\perp}\right)}{\partial E_{n}\left(k_{\perp}\right)}=\sum_{n^{\prime} k_{\perp}^{\prime}} W_{n k_{\perp} \rightarrow n^{\prime} k_{\perp}^{\prime}}\left[f_{n^{\prime}}\left(k_{\perp}^{\prime}\right)-f_{n}\left(k_{\perp}\right)\right]= \\
& =-f_{n}^{A}\left(k_{\perp}\right) \Sigma_{n^{\prime}, k_{\perp}^{\prime}}\left(1-\frac{f_{n^{\prime}}^{A}\left(k_{\perp}^{\prime}\right)}{f_{n}^{A}\left(k_{\perp}\right)}\right) W_{n k_{\perp} \rightarrow n^{\prime} k_{\perp}^{\prime}} \approx \\
& \approx-f_{n}^{A}\left(k_{\perp}\right) \Sigma_{n^{\prime}, k_{\perp}^{\prime}} W_{n k_{\perp} \rightarrow n^{\prime} k_{\perp}^{\prime}} .
\end{aligned}
$$

In the present work, we use the simple qualitative approach that operates with $k_{\perp}=0$ wave functions to calculate electron relaxation times and mobilities for different scattering mechanisms. This approach neglects effects of $s-p$ hybridization. Nevertheless, the $k_{\perp}=0$ 
wave functions correctly describe many principal features of the band structure under consideration, particularly, they correctly describe the localization of electrons in the well and selection rules for interband transitions. Moreover, the energy spectra obtained in terms of the envelope functions approach [17] behave in the same way as such spectra obtained in terms of the $8 \times 8 k \times p$ method [37]. In our calculations, we use nonparabolic dispersion law in order to describe correctly the energy dependence of the density of states. Our approach allows us to calculate and analyze the transition matrix elements for all the considered scattering mechanisms.

\section{Two-dimensional electron relaxation on LO phonons}

Accounted in the calculations of this chapter is only one scattering mechanism - scattering by longitudinal optical phonons. This mechanism was mentioned above as one of the dominant at the liquid nitrogen temperatures for the bulk crystals of MCT. We calculated relaxation times of two-dimensional electrons to reveal the influence of changes in parameters of the QW on the electron scattering processes. In a strict sense, we cannot introduce the momentum-scattering time for inelastic processes. But the relaxation time can be considered to be a good qualitative estimate for the momentum relaxation time, since phonon emission and absorption result in large changes in the electron momentum.

The momentum relaxation time $\tau_{n}\left(k_{\perp}\right)$ of localized electrons can be introduced from [34] and Eq. (2.4) by means of the momentum relaxation rate for electron gas excited initially into the $\left(n, \vec{k}_{\perp}\right)$ state:

$$
\frac{1}{\tau_{n}\left(k_{\perp}\right)}=\sum_{n^{\prime}, k_{\perp}^{\prime}} \frac{k_{\perp}-k_{\perp}^{\prime} \cos \theta}{k_{\perp}} W_{n k_{\perp} \rightarrow n^{\prime} k_{\perp}^{\prime}}
$$

where $\theta$ is the angle between the initial and final electron wave-vectors in the process of scattering.

In [35] it was also proved that the relaxation time approach could be used when phonon energy is higher than the thermal energy $k_{B} T$. Gelmont et al. compared relaxation-time approximation and Monte-Carlo simulations for polar-optical phonon scattering in $\mathrm{GaN}$ and obtained a good agreement between these techniques. Comparison between relaxation-time approximation and Monte-Carlo simulation of electron mobility in $\mathrm{Hg}_{1-\mathrm{x}} \mathrm{Cd}_{\mathrm{x}} \mathrm{Te}$ at liquid nitrogen temperatures for polar-optical phonon scattering was made in [36], also a good agreement was obtained.

For 2D case for steady-state transport under an uniform electric field, we have $\partial f / \partial t=0, \vec{\nabla}_{r} f=0$. For low-field $f^{A}<<f^{S}, f_{S} \approx f$, and the asymmetric part of the occupancy function can be found from Eq. (2.4) and Eq. (3.1):

$$
f_{n}^{A}=\tau_{n}\left(\vec{k}_{\perp}\right) \frac{e}{\hbar} \vec{F} \vec{\nabla}_{\vec{k}} f_{n}
$$

For the longitudinal optical phonon scattering, transition probabilities $W_{n k_{\perp} \rightarrow n^{\prime} k_{\perp}^{\prime}}$ can be written from Fermi's golden rule:

$W_{n k_{\perp} \rightarrow n^{\prime} k_{\perp}^{\prime}}=\frac{2 \pi}{\hbar}\left|\left\langle n k_{\perp}\left|H_{e-p h}\right| n^{\prime} k_{\perp}^{\prime}\right\rangle\right|^{2} \times$

$\times \delta\left[E_{n}\left(k_{\perp}\right)-E_{n^{\prime}}\left(k_{\perp}^{\prime}\right) \mp \hbar w_{L O}\right]$

Where $H_{e-p h}$ is the Hamiltonian of the electron-phonon interaction, and $\hbar w_{L O}$ is the phonon energy.

Detailed calculations of the transition probabilities and matrix elements for the longitudinal optical phonon scattering were carried in [34]. To calculate relaxation times for this scattering mechanism, we use the final formula (6.141) from [34] where the states occupancy in the QW is taken into account:

$1 / \tau_{n}\left(k_{\perp}\right)=\frac{2 e^{2} w_{L O}}{L}\left(\frac{1}{\varepsilon_{\infty}}-\frac{1}{\varepsilon_{0}}\right) \times\left(N_{L O}(T)+\frac{1}{2} \pm \frac{1}{2}\right) \times$

$\times \sum_{n^{\prime}, m}\left\{G\left(n, n^{\prime}, m\right) \times\right.$

$\left.\times \int d^{2} q \cdot\left(1-f_{n^{\prime}}\left(\vec{k}_{\perp}+\vec{q}\right)\right) \frac{\delta\left[E_{n}\left(\vec{k}_{\perp}\right)-E_{n^{\prime}}\left(\vec{k}_{\perp}+\vec{q}\right) \pm \hbar w_{L O}\right]}{\left(q^{2}+(\pi m / L)^{2}\right)}\right\}$,

where

$$
\varepsilon_{0}=20.5-15.6 x+5.7 x^{2}
$$

$\varepsilon_{\infty}=15.2-15.6 x+8.2 x^{2}$ are static and high-frequency dielectric permittivities [27, p. 126], $N_{L O}(T)=1 /\left(\exp \left[\frac{\hbar w_{L O}}{k_{B} T}\right]-1\right)$ is the concentration of LO phonons at a given temperature, $m$ is the quantum number of the phonon mode, $L$ is the $\mathrm{QW}$ width, $\mathrm{n}$ and $n$ ' are the quantum numbers of initial and final electron levels during the scattering process, $\vec{k}_{\perp}$ is the initial wave-vector of electron in the layer plane of the QW.

In $\mathrm{HgCdTe}$ compounds, two types of longitudinal optical phonons exist the first one is related to HgTe and the other one - to CdTe matrices. These phonons have slightly different energies $-\hbar w_{L O 1}=17.1 \mathrm{meV}$ and $\hbar w_{L O 2}=20.8 \mathrm{meV}$, respectively [27]. In our calculations, we assume that the relative concentration of HgTe-related phonons is proportional to $(1-x)$, while the concentration of CdTe-related phonons is proportional to $x$. Relaxation rates in Eq. (3.4) should be calculated for each of these types separately, and then the summary rate should be found from the relation

$1 / \tau_{n}\left(k_{\perp}\right)=1 / \tau_{n}^{L O 1}\left(k_{\perp}\right) \times(1-x)++1 / \tau_{n}^{L O 2}\left(k_{\perp}\right) \times x$

One should note that the relaxation time in Eq. (3.4) depends on the electron kinetic energy via wavevector $\vec{k}_{\perp}$. This relaxation time determines relaxation of the population of the state $\left(n, \vec{k}_{\perp}\right)$. 
In equation (3.4), $G\left(n, n^{\prime}, m\right)$ is the square of the overlap integral for electron and phonon wave functions:

$$
G\left(n, n^{\prime}, m\right) \times=\left(\frac{2}{L} \int_{-L / 2}^{L / 2} d z \cdot \varphi_{n}(z) \cdot \varphi_{n^{\prime}}(z) \cdot \varphi_{p h}(z)\right)^{2}
$$

where $\varphi_{n}(z)$ and $\varphi_{n^{\prime}}(z)$ are the envelope wave functions of the initial and final states of the localized electrons in the scattering process and $\varphi_{p h}(z)$ is the wave function of the LO phonon, which describes the symmetry of the phonon electrostatic field:

$$
\varphi_{p h}(z)=\left\{\begin{array}{c}
\cos \left(\frac{\pi m z}{L}\right), m=2 k+1 \\
\sin \left(\frac{\pi m z}{L}\right), m=2 k
\end{array}\right.
$$

The overlap integral $G\left(n, n^{\prime}, m\right)$ describes the parity selection rules in the process of electron scattering. The integrand should be symmetric to get a non-zero value of $G$. The relaxation time in Eq. (3.4) for electrons from a level $n$ takes into account the scattering of these electrons within the level as well as transitions of these electrons to other levels in the QW.

In these structures, the electron ground level always lies below the bottom of the conduction band of the well, while the first level appears inside the band gap at large widths of QW [17]. With the decrease of the QW width, the excited levels climb up, while the ground level goes deeper under the Fermi level. That's why, 2D electron gas of the ground level is always degenerated, and the degree of degeneracy is determined by the energy interval between the ground level and the Fermi one. The average kinetic energy of electrons on the degenerated level is much higher than the classical value $k T$ for $2 \mathrm{D}$ electrons. We calculated how the average kinetic energy of electrons of different levels depends on the QW width, using the classical formula:

$$
\left\langle E_{k}^{n}\right\rangle=\frac{\Sigma_{k} E_{n}(k) f_{n}(k)}{\Sigma_{k} f_{n}(k)} .
$$

As can be seen from Fig. 3, the ground electron level is always placed below the Fermi level, while the first excited one is placed below it at $L>20 \mathrm{~nm}$. Dependencies of the average electron kinetic energy on the well width $L$ for three lowest levels of the QW are presented in Fig. 4. One can see that the average kinetic energy of the ground level changes from $3.82 \mathrm{kT}$ to $5.34 k T$, while varying the $\mathrm{QW}$ width. The average kinetic energy of the first level varies from $1.18 k T$ to $3.24 k T$ while this energy for the second level is around $1.04 k T$ to $1.13 k T$.

Relaxation times for electrons from the bottom of three ground levels of the QW are presented in Fig. 5(a) (higher levels are not considered because the level of their occupancy is negligible). Relaxation times for electrons with an average kinetic energy at these levels are presented in Fig. 5(b).

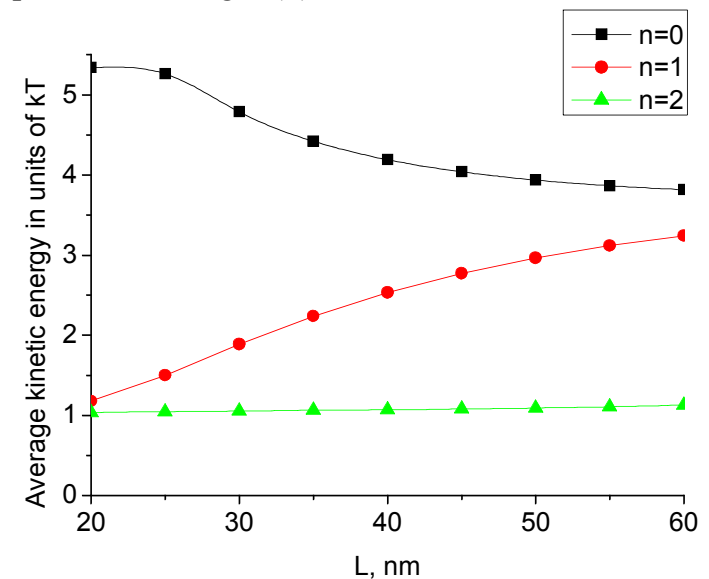

Fig. 4 Dependencies of the average kinetic energies of electrons at the ground $(n=0)$, first $(n=1)$ and second $(n=2)$ levels of $\mathrm{CdTe} / \mathrm{Hg}_{1-\mathrm{x}} \mathrm{Cd}_{\mathrm{x}} \mathrm{Te} / \mathrm{CdTe} \mathrm{QW}$ on the well width $L$. The temperature is taken to be $\mathrm{T}=77 \mathrm{~K}$, the composition $x=0.12$. $E_{C}=0$ is the bottom of the conduction band and $\mathrm{E}_{\mathrm{V}}=-76.6 \mathrm{meV}$ - top of the valence band in the well.

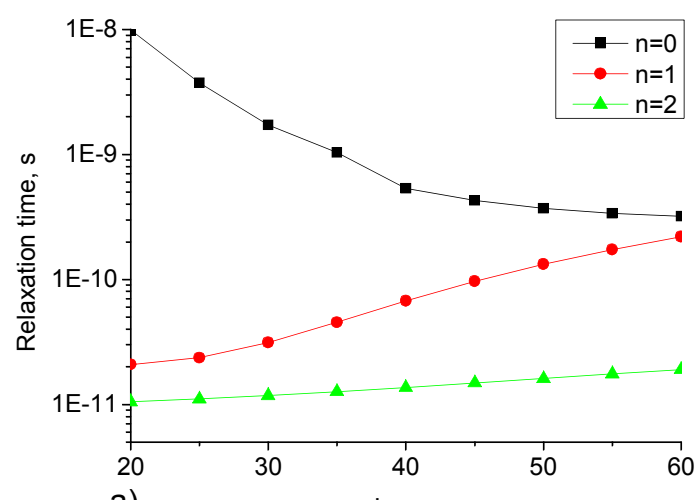

a) $\quad \mathrm{L}, \mathrm{nm}$

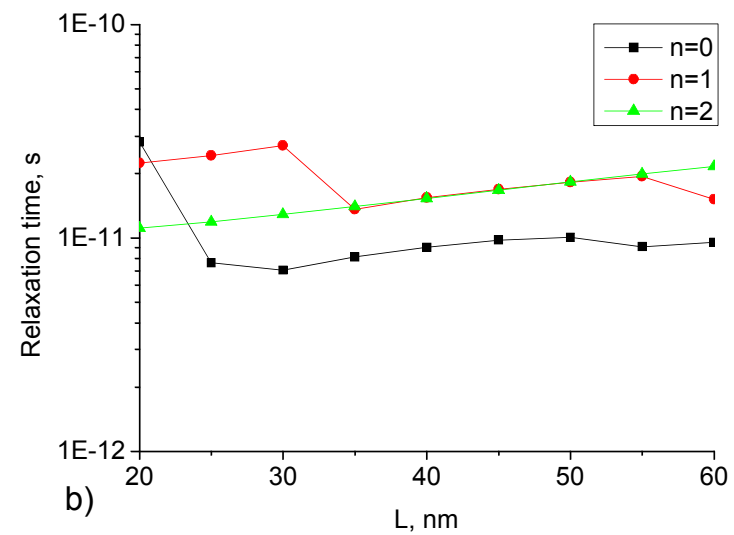

Fig. 5. LO phonon scattering dependences for relaxation times of electrons with zero in-plane kinetic energy from the ground $(n=0)$, first $(n=1)$ and second $(n=2)$ levels (a) and relaxation times of electrons with the average in-plane kinetic energy from these levels (b) in the $\mathrm{CdTe} / \mathrm{Hg}_{1-\mathrm{x}} \mathrm{Cd}_{\mathrm{x}} \mathrm{Te} / \mathrm{CdTe} \mathrm{QW}$ on the well width $L$. Temperature $\mathrm{T}=77 \mathrm{~K}$, composition $x=0.12$. 
The relaxation time of electrons from the bottom of the ground level grows considerably when QW width decreases (see Fig. 5 (a)). Its increase is caused by the growth of the degree of degeneracy of the ground miniband,which leads to decreasing the number of free states where electrons with low kinetic energies can scatter to. Not only the ground, but the first miniband becomes degenerate at large QW widths, when this miniband descends, below the bottom of the conduction band of the well. However, degeneracy of the first level disappears at the small QW widths, when this level is high (see Fig. 3). All other 2D levels at Fig. 3 are nondegenerate.

The drift mobility of electrons in the QW in the applied in-plane electric field $\vec{F}$ can be calculated by averaging all the possible electron velocities in this QW. Taking into account the quantization of the wave-vector $\mathrm{k}$ along the QW axis and using Eq. (3.2), we can rewrite the latter equation:

$$
\begin{gathered}
\mu_{L O}=\frac{1}{|\vec{F}|} \frac{\sum_{\vec{k}} \frac{\hbar \vec{k}}{m^{*}} f_{A}(k)}{\sum_{\vec{k}} f_{A}(k)}=\frac{\Sigma_{n} \mu_{n}^{L O} \int_{\vec{k}_{\perp}} f_{n} \cdot d^{2} \vec{k}_{\perp}}{\sum_{n} \int_{\vec{k}_{\perp}} f_{n} \cdot d^{2} \vec{k}_{\perp}}= \\
=\frac{1}{|\vec{F}|} \frac{\frac{e}{\hbar} \Sigma_{n} \int_{\vec{k}_{\perp}} \tau_{n}\left(\vec{k}_{\perp}\right) \frac{\hbar \vec{k}_{\perp}}{m_{n}\left(k_{\perp}\right)}\left(\vec{F} \cdot \vec{\nabla}_{\vec{k}} f_{n}\right) \cdot d^{2} \vec{k}_{\perp}}{\Sigma_{n} \int_{\vec{k}_{\perp}} f_{n} \cdot d^{2} \vec{k}_{\perp}},
\end{gathered}
$$

where relaxation times are given in Eq. (3.4); effective masses and distribution function depend on the level number $n$ and the modulus of the in-plane wave-vector $k_{\perp}$.

High level of degeneracy and high average kinetic energies of electrons of ground and first levels (at large well widths) lead to the substantial contribution of lateral transitions to the processes of electron scattering on LO phonons. During this lateral transition, electrons with huge kinetic energies are scattered to the levels energy change to which from the initial level is much higher than the phonon energy.

\section{Electron mobility for charged impurities scattering}

We calculated the mobility of electrons on charged impurities in the QW $\mathrm{CdTe} / \mathrm{Hg}_{1-\mathrm{x}} \mathrm{Cd}_{\mathrm{x}} \mathrm{Te} / \mathrm{CdTe}$ by modifying the approach of [18], where it is used the approximation $T=0 \mathrm{~K}$, which allows to get simplifications in the calculations. Here, it is considered more general case. Using Fermi's golden rule and the principle of detailed balance, one can obtain:

$$
\begin{aligned}
& W_{n k_{\perp} \rightarrow n^{\prime} k_{\perp}^{\prime}}=W_{n^{\prime} k_{\perp}^{\prime} \rightarrow n k_{\perp}}=\frac{2 \pi}{\hbar} \delta\left(E_{n}\left(k_{\perp}\right)-E_{n^{\prime}}\left(k_{\perp}^{\prime}\right)\right) \times \\
& \times\left|\left\langle n k_{\perp}\left|H_{d e f}\right| n^{\prime} k_{\perp}^{\prime}\right\rangle\right|^{2} .
\end{aligned}
$$

We will find the solutions of the equations (2.4) and (4.1) in the form of the equations (2.3), (3.2). It is shown in [18] that Eq. (2.3) will be the solution of Eq. (2.4), if relaxation times fulfill the linear equation [18]:

$-\Sigma_{k_{\perp}} \frac{k_{\perp}^{2}}{m_{i}} \frac{\partial f_{i}^{(0)}}{\partial E_{k_{\perp}}}=\Sigma_{j} K_{i j} \tau_{j}$.

We will deal especially with elastic scattering, because scattering by charged impurities changes only the direction of the electron momentum, while the magnitude of this momentum remains unchanged. Transitions between different levels during the scattering process are restricted under this assumption. Consequently, coefficients $K_{i \neq j}$ will be equal to zero, and Eq. (4.2) will be separated by several independent equations for each energy level.

To calculate the relaxation time, we introduce the additional wave-vector $\vec{k}_{\perp}^{\prime}-\vec{k}_{\perp}=\vec{q}_{\perp}$. From the momentum conservation law, we obtain: $\vec{k}_{\perp} \cdot \vec{q}_{\perp}=-2 k_{\perp}^{2} \sin ^{2} \frac{\theta}{2}=-k_{\perp}^{2}(1-\cos \theta)$, where $\theta$ is the angle between the initial and final wave-vectors of electron in the scattering process. We obtain the formula for the relaxation time on electrons of the $n$-th level scattered by charged impurities:

$$
\begin{aligned}
& \frac{1}{\tau_{n}}=-\frac{s m_{n}}{\hbar^{3} 2 \pi} \frac{1}{\int\left[E_{n}-E_{n 0}\right] \frac{\partial f_{n}^{(0)}}{\partial E_{n}} d E_{n}\left(k_{\perp}\right)} \times \\
& \times \iint\left\{\left\langle\left.\left\langle n k_{\perp}\left|H_{d e f}\right| n k_{\perp}+q_{\perp}\right\rangle\right|^{2}\right\rangle_{\text {average }}^{q_{\perp}=q_{\perp}^{0}} \times\right. \\
& \left.\times\left[E_{n}-E_{n 0}\right](1-\cos \theta) \frac{\partial f_{n}^{(0)}}{\partial E_{n}}\right\} d E_{n}\left(k_{\perp}\right) d \theta,
\end{aligned}
$$

where $q_{\perp}^{0}=2 k_{\perp}\left|\sin \frac{\theta}{2}\right|$. One should note that in the latter formula $\partial f_{n}^{(0)} / \partial E_{n}$ is not the Dirac delta function as it was at $T=0$. To find the matrix element $\left\langle\left|\left\langle n k_{\perp}\left|H_{\text {def }}\right| n k_{\perp}+q_{\perp}\right\rangle\right|^{2}\right\rangle_{\text {average }}^{q_{\perp}=q_{\perp}^{0}}$, we follow the same procedure as that described in [18] for $T=0$. However, we modify the screening function that describes the screening in 2DEG to account the non-zero temperature. According to the semi-classical approach, when $q_{\perp} \rightarrow 0$ $[18$, p. 210$]$, the screening function can be found as:

$\xi\left(q_{\perp}\right)=1+q_{0} / q_{\perp}$

where $\quad q_{0}=\frac{2 \pi e^{2}}{\varepsilon_{0}} \frac{\partial n_{e}}{\partial E_{F}} \quad$ and $\quad n_{e}(\vec{r})=\frac{2}{s} \Sigma_{k_{\perp}} \times$ $\times\left\{1+\exp \left[\left(E_{n}-E_{F}\right) / k_{B} T\right]\right\}^{-1}$. Evaluating the derivative of the electron concentration for non-zero temperature, we obtain the final equation for the screening function: 
$\xi\left(q_{\perp}\right)=1+\frac{1}{q_{\perp}} \frac{2 e^{2} m_{n}}{\varepsilon_{0} \hbar^{2}} f_{n}(0)$.

In the limiting case of $T=0 \mathrm{~K}, f_{n}(0)=1$, and Eq. (4.5) reduces to appropriate formula from [18]. Having the explicit Eq. (4.5) for the $\xi\left(q_{\perp}\right)$ and substituting the formula for the matrix element from [18] into Eq. (4.3), we obtain the final formula for the relaxation time of the localized electrons on the $n$-th level, which are scattered by charged impurities at non-zero temperature:

$$
\begin{aligned}
& \frac{\hbar}{\tau_{n}}=-\frac{m_{n}}{\pi \hbar^{2}} \sum_{l} c_{l} \frac{1}{\int\left[E_{n}-E_{n 0}\right] \frac{\partial f_{n}^{(0)}}{\partial E_{n}} d E_{n}\left(k_{\perp}\right)} \times \\
& \times \int d E_{n}\left(k_{\perp}\right)\left[E_{n}-E_{n 0}\right] \frac{\partial f_{n}^{(0)}}{\partial E_{n}} \times \\
& \times \int_{0}^{\pi} d \theta(1-\cos \theta)\left[\frac{2 \pi e Z_{l} e_{l}}{\varepsilon_{0}\left[2 k_{\perp} \sin \frac{\theta}{2}+q_{0}\right]}\right]^{2} \times \\
& \times \int_{z_{\max }^{l}}^{l} d z_{i} g_{\text {imp }}^{2}\left[2 k_{\perp} \sin \frac{\theta}{2}, z_{i}, n\right] .
\end{aligned}
$$

The concentration of $l$-th impurity species (charge $\left.e_{l} Z_{l}\right)$ is denoted by $c_{l}$, and the length over which these impurity species are found in the heterostructure is denoted by $L_{z}^{l}=z_{\max }^{l}-z_{\min }^{l}$. The sum is carried out over all kinds of the charged impurities in the system. For $\mathrm{CdTe} / \mathrm{Hg}_{1-\mathrm{x}} \mathrm{Cd}_{\mathrm{x}} \mathrm{Te} / \mathrm{CdTe} \mathrm{QW}$, one should use the value $Z=1$ or 2 , having in mind $\mathrm{Hg}^{+}$interstitials in $n$ type material or $\mathrm{Hg}^{2-}$ in $p$-type material $[38,39]$.

Distribution of the charged impurities in the system is described by the form-factor $g_{\text {imp }}$ :

$$
g_{\text {imp }}\left(q_{\perp} \cdot z_{i}, n\right)=\int_{-\infty}^{\infty} \chi_{n}^{2}(z) \cdot e^{-q_{\perp}\left|z-z_{i}\right|} d z
$$

where $\chi_{n}(z)$ is the envelope wave function of the $n$-th level electrons. The mobility of electrons for each of these levels can be expressed as:

$\mu_{n}=\frac{e}{m_{n}} \tau_{n}\left(E_{F}\right)$

The average mobility of electrons for only one scattering mechanism (charged impurities scattering) can be found then as:

$$
\mu_{C I}=\frac{\sum_{n} \mu_{n}^{C I} \int_{\vec{k}_{\perp}} f_{n} d^{2} \vec{k}_{\perp}}{\sum_{n} \int_{\vec{k}_{\perp}} f_{n} d^{2} \vec{k}_{\perp}}
$$

In the $n$-type QW with the composition $x=0.12$ and the concentration of background charged impurities $10^{14}-10^{15} \mathrm{~cm}^{-3}$, the Fermi level varies from 5.3 up to $11.2 \mathrm{meV}$ (see Fig. 3). According to our estimations, background impurities affect electron scattering much stronger than the remote delta-doped layer of the equivalent charge density. Thus, background charged impurities play an important role in the scattering for these QWs. In further calculations, we will treat the case of the background impurities scattering, because these impurities are always present in the QW.

\section{Estimation of minor scattering mechanisms}

\subsection{Scattering by acoustic phonons}

In $\mathrm{HgCdTe}$ quantum wells can exist two channels of electron relaxation via the acoustic phonons scattering. These are scattering by the deformation potential interaction mechanism and scattering by the piezoelectric interaction mechanism [34]. However, for the quantum wells, growth direction of which is [001] axis, the piezoelectric interaction is absent [40, p. 48]. So, in this chapter, we will estimate the relaxation time of electrons that scatter on acoustic phonons via deformation potential interaction.

In layered heterostructures, acoustic waves consist of extended and confined modes. Extended modes propagate through the whole heterostructure in any direction. Confined modes are localized in the layer plane of the well and propagate along this plane. However, due to the small differences between elastic properties of the matrix and quantum well materials, this confinement is weak. Thus, confined modes can be neglected, while extended modes can be approximated by plane waves. The energy of acoustic phonon can be estimated as $E_{\text {acoustic }} \sim \hbar s_{L}\left(\frac{2 \pi}{L}\right) \quad[34,6.135]$, where $s_{L}=10^{5} \mathrm{~cm} / \mathrm{s}$ is the sound velocity in the layer plane. For $L=20 \mathrm{~nm}$, the acoustic phonon energy is two orders of magnitude lower than the mean kinetic energy of the ground level electrons (see Fig. 4). Therefore, the scattering can be assumed to be elastic.

Thus, for estimations we used the simple model $[34,6.137]$ that assumes the well to be infinitely deep, acoustic phonons to be bulk-like and non-parabolicity of the dispersion law to be neglected:

$$
\begin{aligned}
& \frac{1}{\tau_{s}\left(n_{0}, k_{0}\right)}=\frac{2(C-a)^{2} m^{*} k_{B} T}{\hbar^{3} s_{L} C_{L} L} \times \\
& \times \Sigma_{n}\left(1+\frac{1}{2} \delta_{n 0, n}\right)\left(1-f_{n 0}\left(k_{0}\right)\right),
\end{aligned}
$$

where $\left(1-f_{n 0}\left(k_{0}\right)\right)$ is the factor that describes the occupancy of the state electron scatters to; assuming the scattering to be elastic [34], we can state $f_{n}(k)=f_{n 0}\left(k_{0}\right)$. The deformation potential constant $(C-a)_{\mathrm{HgCdTe}}=$ $-3.63 \mathrm{eV}$ is (see Eq. (1.1)); $m^{*}$ is the electron effective 
mass, $n_{0}$ and $n$ are the numbers of electron levels and $C_{L}=M_{0} s_{L} / V_{0}=5.0625 \times 10^{17} \mathrm{eV} \cdot \mathrm{s} / \mathrm{cm}^{4}$ is the mean elastic constant of the material (taken to be the same as in $\mathrm{HgTe}), M_{0}$ and $V_{0}$ are the mass and the volume of the primitive cell of $\mathrm{HgTe}$.

According to our estimates, the relaxation time of the ground level electrons with the average kinetic energy is close to $6 \times 10^{-10} \mathrm{~s}$ for $L=20 \mathrm{~nm}$ and $3.8 \times 10^{-10}$ $\mathrm{s}$ for $L=60 \mathrm{~nm}$; the relaxation time of first level electrons with the average kinetic energy is around $2.6 \times 10^{-10} \mathrm{~s}$ for $L=60 \mathrm{~nm}$. These times are much larger than appropriate times for longitudinal optical phonon scattering (see Fig. 5 (b)). Consequently, the acoustic phonons scattering is small and can be neglected in the further treatment.

\subsection{Scattering on interface roughness}

Very little is known about the microscopic structure of the interface defects so they usually use very simple models for the calculation of scattering by these defects. All estimations are carried out for the well of the 20-nm width, which is the minimal width presented in our calculations.

To estimate the scattering by interface roughness, we use the model presented in the book of Bastard [18] In this model, the interface defect is assumed to have the thickness $h$ of one monolayer in the growth direction and have extensions $l_{x}$ and $l_{y}$ in the layer plane. It is assumed that these defects are located far away from each other, so scattering events on different defects are independent from each other. Calculations are made at $T=0 \mathrm{~K}$ in the Electric Quantum Limit, which assumes that only the lowest electron sub-band is occupied. In our system, at small well widths electrons scatter from the ground level, therefore this assumption is reasonable. We used the approach from [18], which allows to take into account the sharpness of the electron envelope function at the interface:

$$
\begin{aligned}
& \frac{1}{\tau_{\text {roug }}\left(E_{F}\right)}=16 h^{2} V_{b}^{2} N_{d e f} \frac{m_{0}}{\pi \hbar^{3}}\left(\int_{0}^{h} \chi_{0}^{2}(z) d z\right) \times \\
& \times \int_{0}^{\pi} \frac{d \theta}{\xi^{2}\left(2 k_{F} \sin \frac{\theta}{2}\right)} \frac{\sin ^{2}\left[\frac{k_{F} l_{x}}{2}(\cos \theta-1)\right] \sin ^{2}\left[\frac{k_{F} l_{y}}{2} \sin \theta\right]}{k_{F}^{4} \sin ^{2}[\theta](1-\cos \theta)},
\end{aligned}
$$

where $k_{F}$ is the Fermi wave vector, $\chi_{0}(z)-$ envelope wave function of the ground level electrons, $V_{b}$ - barrier height, $m_{0}$ - effective mass of the ground level electrons, $\xi\left(k_{\perp}\right)$ is defined in Eq. (4.5), $\theta$ is the angle between electron wave vectors before and after the scattering.

To estimate the relaxation rate from the latter equation, one should know the average concentration of interface defects $N_{\text {def }}$ and their average lateral dimensions $l_{x}$ and $l_{y}$. Lateral dimensions of the defects were taken from [41], which is often used for estimations of the interface scattering in quantum wells. We have found no data about the concentration of interface roughness defects in $\mathrm{HgCdTe}$ QWs. Nevertheless, the authors [42] created $\mathrm{HgCdTe}$ heterostructures with fixed charges at the interfaces with the concentration within the range $10^{10}-5 \times 10^{10} \mathrm{~cm}^{-2}$. We assume that the concentration of roughness defects $N_{\text {def }}$ is the same as the concentration of interface fixed charges in the samples of [42]. Thus, we obtain that for ground level electrons with the average kinetic energy, the relaxation time for interface scattering in the well of 20 -nm width is close to $10^{-9}-7 \times 10^{-9} \mathrm{~s}$. This relaxation time is an order of magnitude higher than the appropriate time for the longitudinal optical phonon scattering (see Fig. 5 (b), thus the interface scattering can be considered as negligible in the further treatment.

\subsection{Influence of the value of the valence-band offset}

Values of the valence-band offset $\Delta$ in the literature are contradicting (see section 1.1). Then, it is important to estimate the influence of variation of this parameter on the mobility of localized electrons. We compared the mobilities for charged impurities scattering and for the LO phonons scattering for two QW widths - 20 and 60 $\mathrm{nm}$. There are the values compared as to the valenceband offset $\Delta_{1}=0.55 \times(1-x) \mathrm{eV}$ and $\Delta_{2}=0.35 \times(1-x) \mathrm{eV}$. According to our calculations, different values of the valence-band offset lead to the mobilities that differ by approximately 1.5 times at small well widths for each of the scattered mechanisms. For the wide wells, variation of the mobility with the change in the valence-band offset is weaker.

Thus, we can see that the uncertainty in the value of the valence-band offset strongly affects the magnitude of the electron mobilities, and it is principally impossible to obtain precise numerical results for relaxation times and mobilities. Also, it is impossible to estimate the strain values precisely, because they depend on the other technological heterostructure layers and on the heterostructure growth conditions.

You can also aim the reduction of stress in the layers. Our calculations show that for the QW with the concentration of $n$-type charged impurities of $10^{15} \mathrm{~cm}^{-3}$, the value of strain sufficiently affects the mobility. For example, in the well of $20-\mathrm{nm}$ width, the mobility in the absence of strains is in times greater than such mobility in the presence of the strain (see section 6). Thus, the absence of the strain leads to growth in the electron mobility.

\section{Results for electron mobility in $\mathrm{CdTe} / \mathrm{Hg}_{1-\mathrm{x}} \mathrm{Cd}_{\mathrm{x}} \mathrm{Te} / \mathrm{CdTe}$ quantum well}

The average mobility of electrons in the $\mathrm{CdTe} /$ $\mathrm{Hg}_{1-\mathrm{x}} \mathrm{Cd}_{\mathrm{x}} \mathrm{Te} / \mathrm{CdTe} \mathrm{QW}$ that includes both the LO phonons scattering (Eq. (3.9)) and charged impurities scattering (Eq. (4.9)) can be found as: 


$$
\langle\mu\rangle=\frac{\Sigma_{n}\langle\mu\rangle \int_{\vec{k}_{\perp}} f_{n} \cdot d^{2} \vec{k}_{\perp}}{\Sigma_{n} \int_{\vec{k}_{\perp}} f_{n} \cdot d^{2} \vec{k}_{\perp}},
$$

where the summary mobility of the $n$-th level electrons is found from the relation

$$
1 /\left\langle\mu_{n}\right\rangle=1 / \mu_{n}^{L O}+1 / \mu_{n}^{C I}
$$

Results for the electron mobility for LO-phonon scattering only (Eq. (3.9)) and results for the average mobility that includes two scattering mechanisms (Eq. (6.1)) are plotted in Fig. 6.

The electron mobility that takes into account only the LO phonons scattering, grows substantially, when the QW width decreases. Therefore, at small charged impurity concentrations the LO phonons scattering dominates, and the average electron mobility also grows at small well widths. When the concentration of charged impurities is greater, the growth of average mobility is partially suppressed. For example, for the concentration of $n$-type charged impurities $10^{16} \mathrm{~cm}^{-3}$, the average mobility grows approximately two times only. These results that describe variation of the average electron mobility with the quantum well width can be possibly used to optimize growth procedure for $\mathrm{CdTe} / \mathrm{Hg}_{1-\mathrm{x}} \mathrm{Cd}_{\mathrm{x}} \mathrm{Te} / \mathrm{CdTe} \mathrm{QWs}$ with different properties within the same technological line.

It is important to note that usage of more pure samples of $\mathrm{Hg}_{1-\mathrm{x}} \mathrm{Cd}_{\mathrm{x}} \mathrm{Te}$ with lower concentrations of charged impurities allows one to obtain higher electron mobilities for the charged impurities scattering. In this case, the average electron mobility also is higher. Also, one should note that in this paper we considered the model sample where strain is not relaxed. In the case of using the samples with buffer layers that partially relax the strain, the strain-induced increase of the band gap will be smaller, and the mobility of localized electrons should increase by several times.

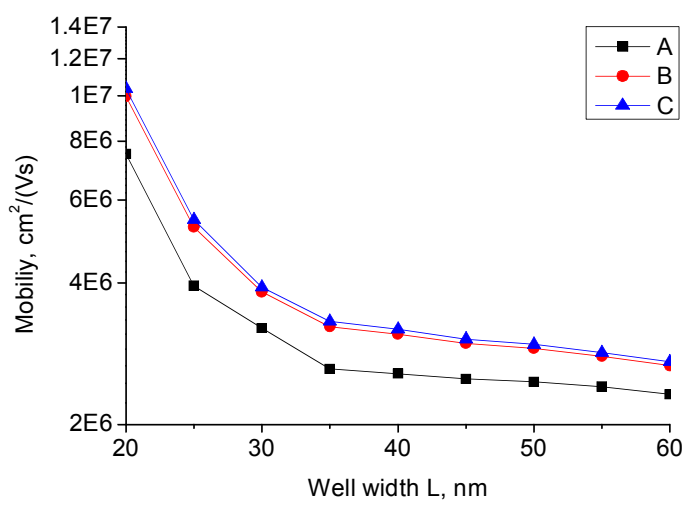

Fig. 6. Electron mobility in the $\mathrm{CdTe} / \mathrm{Hg}_{1-x} \mathrm{Cd}_{\mathrm{x}} \mathrm{Te} / \mathrm{CdTe} \mathrm{QW}$. The mobility includes longitudinal optical phonon scattering (LOP) and charged impurities scattering (CI). Line A is for LOP and CI scattering with the concentration $10^{15} \mathrm{~cm}^{-3}$, line $\mathrm{B}$ is for LOP and CI scattering with the concentration $10^{14} \mathrm{~cm}^{-3}$, line $\mathrm{C}$ is for pure LOP scattering mechanism. The parameters used are as follows: temperature $T=77 \mathrm{~K}$; composition $x=0.12$.
Unfortunately, the authors do not know the experimental data on the mobility of electrons in the $\mathrm{CdTe} / \mathrm{Hg}_{1-\mathrm{x}} \mathrm{Cd}_{\mathrm{x}} \mathrm{Te} / \mathrm{CdTe}$ quantum well with inverted band structure at the nitrogen temperature. Therefore, it is useful to compare electron mobilities in the $\mathrm{CdTe} / \mathrm{Hg}_{1-\mathrm{x}} \mathrm{Cd}_{\mathrm{x}} \mathrm{Te} / \mathrm{CdTe} \mathrm{QW}$ with inverted band scheme in the well (Fig. 6) with electron mobilities in other $\mathrm{Hg}_{1-\mathrm{x}} \mathrm{Cd}_{\mathrm{x}} \mathrm{Te}$ structures at $T=77 \mathrm{~K}$. The electron mobility in bulk undoped $\mathrm{Hg}_{1-\mathrm{x}} \mathrm{Cd}_{\mathrm{x}} \mathrm{Te}$ with the direct band scheme at the composition $x=0.2$ is $2.5 \times 10^{5} \mathrm{~cm}^{2} /(\mathrm{V} \times \mathrm{s})$ [27, p. 95]. The electron mobility in bulk $\mathrm{HgTe}$ (which has the inverted band scheme) is $2.2 \times 10^{5} \mathrm{~cm}^{2} /(\mathrm{V} \times \mathrm{s})$ [27, p. 95].

According to our calculations, the electron mobility in the QW $\mathrm{CdTe} / \mathrm{Hg}_{1-x} \mathrm{Cd}_{x} \mathrm{Te} / \mathrm{CdTe}$ with the concentration of $n$-type charged impurities $10^{15} \mathrm{~cm}^{-3}$ is not higher than $6-7 \times 10^{5} \mathrm{~cm}^{2} /(\mathrm{V} \times \mathrm{s})$ for the composition $x=0.2$, and it is close to $1-1.2 \times 10^{6} \mathrm{~cm}^{2} /(\mathrm{V} \times \mathrm{s})$ for the composition $x=0$. It was shown in [13] that the electron mobilities in the QW CdTe/HgTe/CdTe (at $T=3 \mathrm{~K}$ ) can reach $2.8 \times 10^{5} \mathrm{~cm}^{2} /(\mathrm{V} \times \mathrm{s})$. From the comparison of these mobilities one can see that the maximal mobility of electrons in $\mathrm{Hg}_{1-\mathrm{x}} \mathrm{Cd}_{\mathrm{x}} \mathrm{Te}$ could be reached in quantum wells with the inverted band scheme inside the well.

One should note that, at very small QW widths (about $10 \mathrm{~nm}$ and less [12]), the width of interfaces becomes comparable with the width of the quantum well. So the scattering by interface roughness [34, p. 249] and the scattering by interface phonons [34, p. 287] become more important and the growth of mobility can be suppressed.

Apparently, we can expect the highest mobility in mercury-cadmium-telluride structures with the inverted band scheme quantum wells at moderate concentrations of charged impurities. Therefore, these structures are of interest for the creation of semiconductor $\mathrm{THz}$ detectors based on hot electrons operating under moderate cooling (up to liquid nitrogen temperature) or ambient temperatures. It is known that narrow gap semiconductors are promising materials for direct $\mathrm{THz}$ detectors due to high electron mobility, high carrier concentration and low effective masses of electrons $[3,43]$. In our case, we should expect the electron relaxation time of about $10^{-11} \mathrm{~s}$ and scattering mean free path of about $50 \mu \mathrm{m}$. For this relaxation time, the QW structure provides a strong interaction with $\mathrm{THz}$ radiation by the free-carrier Drude absorption mechanism [44] in the frequency range of 100-700 GHz. The high conductivity of degenerate electron gas on the metal type structure provides resistance to the order of a few tens of ohms, which will ensure a good matching with the planar metallic antenna. A large mean free path carriers will implement those structures technologically.

Therefore, the quantum wells with the inverted band scheme $\mathrm{HgCdTe}$ are the promising structures for $\mathrm{THz}$ detector design. Thus, it is possible to realize detectors on the effect of the electron gas heating, such as hot electron bolometers or field effect transistors with 
a shallow channel on the basis of the in-plane transport in the quantum well [45].

\section{Conclusions}

We have modelled electron relaxation processes in the ntype $\mathrm{CdTe} / \mathrm{Hg}_{1-\mathrm{x}} \mathrm{Cd}_{\mathrm{x}} \mathrm{Te} / \mathrm{CdTe}$ wide QWs with the inverted band structure at the liquid nitrogen temperatures and for $x$ close to the band inversion value 0.16 , for their potential usage as $\mathrm{THz}$ range detectors. It was found that the longitudinal optical phonons scattering and charged impurities scattering are dominant mechanisms, while the acoustic phonons scattering and interface roughness scattering can be neglected. It was shown that the electron mobility could reach values of the order of magnitude of $5 * 10^{6}-10^{7}$ $\mathrm{cm}^{2} /(\mathrm{Vs})$ for the well widths $60-20 \mathrm{~nm}$ in such structure. These values could be estimated at the concentration of the diluted charged impurity of $10^{14}$ $10^{15} \mathrm{~cm}^{-3}$.

Usage of relaxing buffer layers allows one to reach the partial relaxation of the strains in the quantum well and to increase the mobility by several times. This mobility is an order of magnitude higher than for the QW with the direct band structure and bulk mercurycadmium-telluride. On the one hand, it places high demands on the quality of the structure. On the other hand, it provides the possibility to implement fast direct terahertz detectors, for example, field effect transistors with the shallow channel based on the quantum well or hot electron bolometer operating at moderate cooling.

\section{References}

1. P.H. Siegel, THz technology: An overview // Intern. J. High Speed Electronics and Systems 13(2), p. 351-394 (2003).

2. M.A. Kinch and B.V. Rollin, Detection of millimetre and sub-millimetre wave radiation by free carrier absorption in a semiconductor // Br. J. Appl. Phys. 14, p. 672 (1963).

3. F. Sizov, $\mathrm{THz}$ radiation sensors // Optoelectron. Rev. 18(1), p. 10-36 (2010).

4. K.D. Mynbaev, N.L. Bazhenov, V.I. IvanovOmskii et al., Photoluminescence of $\mathrm{CdHgTe}$ epilayers grown on silicon substrates // Technical Phys. Lett. 36(12), p. 1085-1088 (2010).

5. K.D. Mynbaev, N. L. Bazhenov, V. I. IvanovOmskii et al., Photoluminescence of CdHgTe based nanoheterostructures // Technical Phys. Lett. 36(12), p. 1099-1102 (2010).

6. V.I. Ivanov-Omskii, K.D. Mynbaev, N.L. Bazhenov et al., Optical properties of molecular beam epitaxy-grown $\mathrm{HgCdTe}$ structures with potential wells // Phys. Status Solidi (c), 7(6), p. 1621-1623 (2010).

7. S. Dvoretsky, N. Mikhailov, Yu. Sidorov, V. Shvets, S. Danilov, B. Wittman, and S. Ganichev, Growth of HgTe quantum wells for IR to $\mathrm{THz}$ detectors // J. Electron. Mater. 39(7), p. $918-923$ (2010).

8. M. Pociask, I.I. Izhnin, K.D. Mynbaev, A.I. Izhnin, S.A. Dvoretsky, N.N. Mikhailov, Yu.G. Sidorov, V.S. Varavin, Blue-shift in photoluminescence of ion-milled $\mathrm{HgCdTe}$ films and relaxation of defects induced by the milling // Thin Solid Films, 518(14), p. 3879-3881 (2010).

9. D.A. Kozlov, Z.D. Kvon, N.N. Mikhailov, S.A. Dvoretsky, J.C. Portal, Cyclotron resonance in a two-dimensional semimetal based on a $\mathrm{HgTe}$ quantum well // Pis'ma v ZhETF, 93(3), p. 186-189 (2011), in Russian..

10. E.H. Hwang, S. Das Sarma, Limit to twodimensional mobility in modulation-doped GaAs quantum structures: How to achieve a mobility of 100 million // Phys. Rev. B, 77(23), 235437 (2008).

11. J.R. Meyer, D.J. Arnold, C.A. HofFman, and F.J. Bartoli, Free-carrier transport in superlattices: Smooth transition between the quasi-twodimensional and uniform three-dimensional limits // Phys. Rev. B, 45(3), p. 1295-1304 (1992).

12. J.R. Meyer, D.J. Arnold, C.A. HofFman, F.J. Bartoli, L.R. Ram-Mohan, Electron and hole in-plane mobilities in $\mathrm{HgTe}-\mathrm{CdTe}$ superlattices // Phys. Rev. B, 46(7), p. 4139-4146 (1992).

13. E.B. Olshanetsky, S. Sassine, Z.D. Kvon, N.N. Mikhailov, S.A. Dvoretsky, J.C. Portal, A.L. Aseev, Quantum Hall liquid-insulator and plateau-to-plateau transitions in a high mobility 2DEG in a HgTe quantum well // Pis'ma $v$ ZhETF, 84(10), p. 661-665 (2006), in Russian.

14. J.J. Dubowski, T. Dietl, W. Szymanska, R.R. Gakazka, Electron scattering in $\mathrm{Cd}_{\mathrm{x}} \mathrm{Hg}_{1-\mathrm{x}} \mathrm{Te} / /$ J. Phys. Chem. Solids, 42(5), p. 351-362 (1981).

15. G. Bastard, Theoretical investigations of superlattice band structure in the envelope-function approximation // Phys. Rev. B, 25(12), p. 75847597 (1982).

16. W. Walukiewicz, Electron mobility and thermoelectric power in pure mercury telluride // J. Phys. C: Solid State Phys. 9(10), p. 1945 (1976).

17. E.O. Melezhik, J.V. Gumenjuk-Sichevska, and F.F. Sizov, Simulation of relaxation times and energy spectra of the CdTe / $\mathrm{Cd}_{\mathrm{x}} \mathrm{Hg}_{1-\mathrm{x}} \mathrm{Te} / \mathrm{CdTe}$ quantum well for variable valence band offset, well width, and composition $x$ // Semiconductors, 44(10), p. 1321-1327 (2010).

18. G. Bastard, Wave Mechanics Applied to Semiconductor Heterostructures. Halsted Press, New York, 1988.

19. E.A. Kraut, The effect of a valence-band offset on potential and current distributions in $\mathrm{HgCdTe}$ heterostructures // J. Vac. Sci. Technol. A, 7(2), p. 420-423 (1989).

20. P.M. Hui, H. Ehrenreich, N.F. Johnson, A possible resolution of the valence-band offset controversy in HgTe/CdTe superlattices // J. Vac. Sci. Technol. A 7(2), pp. 424-426 (1989). 
21. M. Truchsess, V. Latussek, C. R. Becker, E. Batke, Temperature dependent investigation of the HgTeCdTe valence band offset // Journal of Crystal Growth 159, pp. 1128-1131 (1996).

22. C.R. Becker, V. Latussek, M. Li, A. PfeufferJeschke, G. Landwehr, Valence band offset in $\mathrm{HgTe} / \mathrm{Hg}_{1-\mathrm{x}} \mathrm{Cd}_{\mathrm{x}} \mathrm{Te}$ superlattices // J. Electron. Mater. 28(6), p. 826-829 (1999).

23. D. Eich, K. Ortner, U. Groh, Z.H. Chen, C.R. Becker, G. Landwehr, R. Fink, E. Umbach, Band discontinuity and band gap of MBE grown $\mathrm{HgTe} / \mathrm{CdTe}(001)$ heterointerfaces studied by kresolved photoemission and inverse photoemission // Phys. Stat. Sol. (a), 173, p. 261-267 (1999).

24. J. Downes, D.A. Faux, Calculation of strain distributions in multiple-quantum-well strainedlayer structures // J. Appl. Phys. 77, p. 2444-2447 (1995).

25. E. Melezhik, O. Korotchenkov, Modeling boundary conditions for computation of piezoelectric fields in quantum dots with image charge analogy // J. Appl. Phys. 102, 013503 (2007).

26. E. Melezhik, O. Korotchenkov, Elastic fields of quantum dots in semi-infinite matrices: Green's function analytical analysis // J. Appl. Phys. 105, 023525 (2009).

27. A.V. Liubchenko, E.A. Salkov, F.F. Sizov, Physical basis of semiconductor infrared photoelectronics (Naukova Dumka, Kyiv, 1984) (in Russian).

28. G.L. Bir, G.E. Pikus, Simmetriya i deformacionnye e'ffekty v poluprovodnikah (Nauka, Moscow, 1972) (in Russian).

29. V. Latussek, C.R. Becker, G. Landwehr, R. Bini and L. Ulivi, Deformation potentials of the semimetal HgTe // PRB 71, pp. 125305-125311 (2005).

30. A.E. Merad, M.B. Kanoun, G. Merad, J. Cibert, H. Aourag, Full-potential investigation of the electronic and optical properties of stressed CdTe and ZnTe // Mat. Chem. Phys. 92(2), pp. 333-339 (2005).

31. E.B. Olshanetsky, Z.D. Kvon, N.N. Mikhailov, E.G. Novik, I.O. Parm, S.A. Dvoretsky, Twodimensional semimetal in HgTe-based quantum wells with surface orientation (100) // Sol. State. Comm. 152(4), pp. 265-267 (2012).

32. Fu. Liang, C. L. Kane, Topological insulators with inversion symmetry // PRB 76(4), pp. 045302045318 (2007).

33. R.V. Goldstein, V.A. Gorodtsov, D.S. Lisovenko, Young's modulus of cubic auxetics // Letters on materials 1(3), pp. 127-132 (in Russian) (2011).
34. V. Mitin, A. Kochelap, A. Stroscio, Quantum Heterostructures: microelectronics and optoelectronics (Cambridge Univercity Press, Cambridge, 1999).

35. B. Gelmont, K. Kim, M. Shur, Monte Carlo simulation of electron transport in gallium nitride // J. Appl. Phys. 74(3), pp. 1818-1821 (1993).

36. Sang Dong Yoo, Kae Dal Kwack, Theoretical calculation of electron mobility in $\mathrm{HgCdTe} / / \mathrm{J}$. Appl. Phys. 81(2), pp. 719-725 (1997).

37. E. G. Novik, A. Pfeuffer-Jeschke, T. Jungwirth, V. Latussek, C.R. Becker, G. Landwehr, H. Buhmann, and L.W. Molenkamp, Band structure of semimagnetic $\mathrm{Hg} 1-\mathrm{yMnyTe}$ quantum wells // Phys. Rev. B 72(3), pp. 035321-035332 (2005).

38. H.G. Robinson, D.H. Mao, B.L. Williams, S. Hollander-Gleixner, J.E. Yu, and C.R. Helms, Modeling ion implantation of $\mathrm{HgCdTe} / / \mathrm{J}$. Electron. Mater. 25(8), p. 1336-1340 (1996).

39. B.L. Williams, H.G. Robinson, C.R. Helms, Ion dependent interstitial generation of implanted mercury cadmium telluride // Appl. Phys. Lett. 71(5), p. 692-694 (1997).

40. M.A. Kinch, Fundamentals of Infrared Detector Materials. SPIE, Washington, 2007.

41. T. Ando, Self-consistent results for a GaAs/ $\mathrm{Al}_{\mathrm{x}} \mathrm{Ga}_{1-\mathrm{x}} \mathrm{As}$ heterojunction. II. Low temperature mobility // J. Phys. Soc. Japan, 51(12), p. 39003907 (1982).

42. V. Ariel, V. Garber, D. Rosenfeld, G. Bahir, V. Richter, N. Mainzer, A. Sher, Electrical and structural properties of epitaxial $\mathrm{CdTe} / \mathrm{HgCdTe}$ interfaces // J. Electron. Mater. 24(9), p. 1169-1174 (1995).

43. V.N. Dobrovolsky, F.F. Sizov, A room temperature, or moderately cooled, fast $\mathrm{THz}$ semiconductor hot electron bolometer // Semicond. Sci. Technol. 22, p. 103-106 (2007).

44. N.A. Kabir, Y. Yoon, J.R. Knab et al., Terahertz transmission characteristics of high-mobility GaAs and InAs two-dimensional-electron-gas systems // Appl. Phys. Lett. 89(13), p. 132109-132111 (2006).

45. F. Sizov, A. Golenkov, D. But, M. Sakhno, V. Reva, Sub- $\mathrm{THz}$ radiation room temperature sensitivity of long-channel silicon field effect transistors // Opto-Electronics Rev. 20(2), p. 194199 (2012). 